https://doi.org/10.15407/ujpe66.5.424

D.V. SLOBODIANIUK

Taras Shevchenko National University of Kyiv

(64, Volodymyrs'ka Str., 01601, Kyiv, Ukraine; e-mail: denslobod@ukr.net)

\title{
EXCITATION OF ULTRASHORT SPIN WAVES VIA SPIN-CHERENKOV EFFECT IN MAGNETIC WAVEGUIDES
}

\begin{abstract}
The excitation of ultrashort wavelength spin waves via the spin-Cherenkov effect in magnetic waveguides is investigated via a micromagnetic modeling. The proposed excitation method is relatively simple and easily tunable. The excitation efficiency of the proposed scheme is obtained for different excitation pulse velocities and widths. A coupled waveguide system is also considered. In this case, the spin waves are excited in the first waveguide and then are transferred to the second one due to the dipolar coupling between waveguides. It is also shown that the excitation and transfer of excited spin waves have some limitations related to the dipolar coupling mechanism between the waveguides.

Ke ywords: spin-Cherenkov effect, magnetic waveguide, spin wave, spintronics.
\end{abstract}

\section{Introduction}

Spin waves (SWs) play a key role as signal carriers for the information processing in the novel field of magnonics. This is due to a low power loss and a possibility to miniaturize future data-processing devices using short-wavelength spin waves. However, finding an efficient method of excitation of the short wavelength spin waves is a challenging task. Typical methods of their excitation use pattern inductive antennas to transform a microwave into SW $[1,2]$ or direct electric current-induced spintransfer torques in nano-contact geometries $[3,4]$. However, the major drawback of these methods is the inability to efficiently generate propagating spin waves with wavelengths below the patterning sizes involved. These limitations were overcome in recent works utilizing a post-excitation variation of the magnonic index $[5,6]$ or Fano resonances $[7,8]$.

The most recent approach for the excitation of ultrashort SWs is to use the driven dynamics of a spin vortex core [9]. As a result of such excitation, the core emits an ultrashort spin wave that is imaged by timeresolved x-ray microscopy. However, this approach is cumbersome and requires a setup difficult to function. In addition, the emitted radiant SWs are not very useful for spintronics applications.

(C) D.V. SLOBODIANIUK, 2021
Another fundamentally different approach to the excitation of spin waves utilizes parametric processes of interaction between the external electromagnetic pumping applied to a sample and spin waves $[17,18]$. This method allows the excitation of spin waves in a wide range of wavevectors. However, it requires the use of external $\mathrm{GHz}$-frequency generators and effective ways to apply the electromagnetic pumping to the sample via a microstrip antenna or open dielectric resonator. The amplitudes of the excited spin waves are bounded from above due to different nonlinearities that arise in the ensemble of excited spin waves [18].

On the other hand, the spin-Cherenkov effect (SCE) provides an effective method of SW excitation. It was first observed during the micromagnetic study of the domain wall propagation in ferromagnetic nanotubes [10]. It was shown that, for this geometry, the Walker breakdown is completely suppressed, and the ensemble of moving domain walls can achieve velocities above $1000 \mathrm{~m} / \mathrm{s}$. However, a new limitation arises, when the domain wall reaches the SW phase velocity, thereby triggering a Cherenkov-like emission of the corresponding spin waves. Later, this effect was investigated in detail in [11], where it was shown that SCE has general nature and can occur for a localized magnetic field pulse moving sufficiently rapidly along the surface of a ferromagnet. Those findings open up the effective tunable mechanism of SW excitation.

ISSN 2071-0194. Ukr. J. Phys. 2021. Vol. 66, No. 5 
In the current work, we will investigate the SW excitation in magnetic waveguides via a moving field pulse with different parameters. We will also consider a system of coupled laterally adjacent waveguides in which the spin waves excited via SCE are transferred due to the dipolar coupling between waveguides.

\section{Results and Discussion}

In this work, we will consider an yttrium iron garnet (YIG) waveguide similar to one proposed in work [12] with the following dimensions: $100 \mathrm{~nm}$ wide, $10 \mathrm{~nm}$ thick, and $L=12 \mu \mathrm{m}$ long. The choice of the length $L$ is governed by the simulation convenience, since the consideration of long waveguide allows us to neglect boundary effects and reflections that happen on the waveguide edges. The waveguide geometry will lead to the excitation of plane spin waves that is convenient for applications. For material parameters, we choose typical YIG parameters that can be found elsewhere [13]. The key features of YIG are a low damping and a smaller value of the saturation magnetization $M_{s}$ compared to metallic materials allowing the longer lifetimes for SWs. To investigate the SCE effect, we will use the micromagnetic modeling with the OOMMF software package [14].

First, we analyze SCE in single YIG waveguides in the presence of the external magnetic field $H_{0}=$ $=100$ Oe along the waveguide length. Thus, we are working in the backward volume magnetostatic wave geometry (BWMSW) [15]. We will investigate SCE in the following excitation model. The out-ofplane magnetic field $H_{p}=10$ Oe will be applied to the center of the waveguide, and the rectangular field pulse with the width $w=10 \mathrm{~nm}$ will propagate with different velocities $v_{\text {pulse }}$ along the waveguide. As a result of SCE, it will excite forward and backward spin waves with different wavevectors, but with equal phase velocities $v_{\text {phase }}=\frac{\omega_{\mathrm{SW}}}{k_{\mathrm{SW}}}$. Here, $\omega_{\mathrm{SW}}$ is the SW frequency, and $k_{\mathrm{SW}}$ is the SW wavevector (see Fig. 1, a). The velocities of excited spin waves match the velocities of the moving field pulse $v_{\text {phase }}=v_{\text {pulse }}$. Thus, by varying the pulse velocity, one can excite different spin waves with different wavevectors. It should be noted that, in the original work [10], SCE was observed as a result of the domain wall motion process in magnetic nanotubes. In our case, similar to [16], we use the "artificial" inhomogeneity region that is used to excite spin waves via SCE (Fig. 1, a). The excitation of forward and
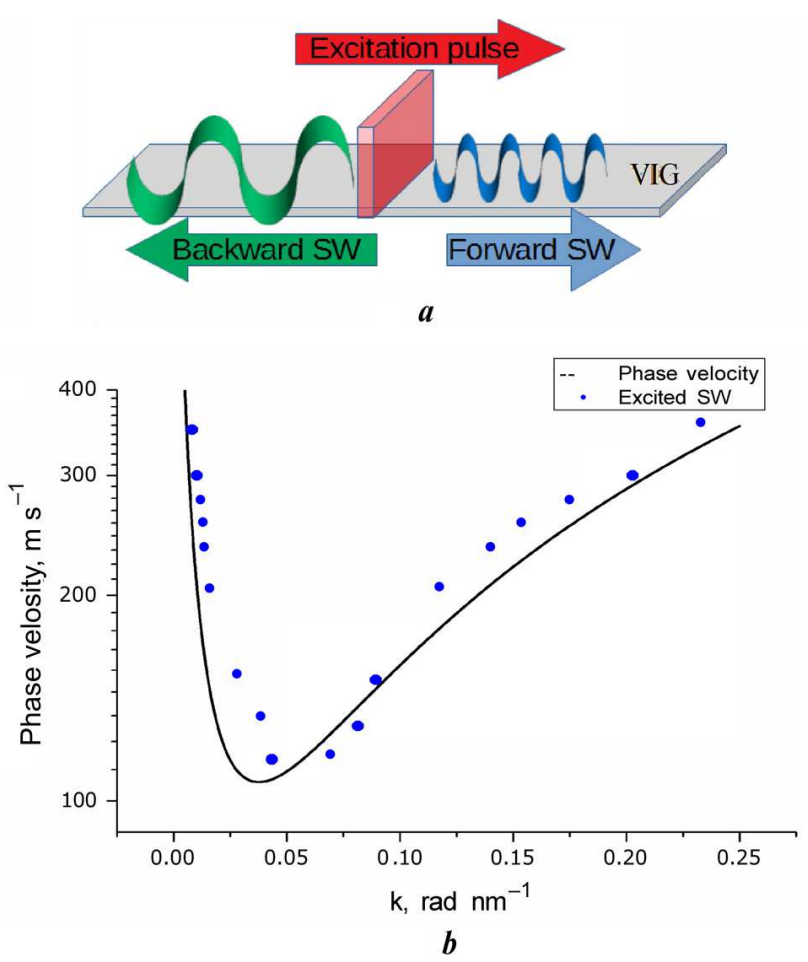

Fig. 1. Schematic diagram showing the model of excitation via SCE in a single YIG waveguide $(a)$, graph showing the modeling results. Solid line - theory, points - modeling for different velocities of the excitation pulse $(b)$

backward SWs is due to the dipole-exchange spectrum of BWMSW. For the given pulse velocity $v_{\text {pulse }}$, two spin-wave modes with different phase velocities $v_{\text {phase }}$ exist. This twofold degeneracy explains the bichromatic excitation of spin waves. Hereinafter, we will mention the phase velocity of spin waves, keeping in mind the fact that the pulse velocity is equal to it.

The solid line in Fig. 1, $b$ shows the theoretically calculated phase velocities of SWs in an YIG waveguide with the parameters described above. We used the theoretical approach from [12] to calculate the SW spectrum and, thus, phase velocities. Varying the velocity of the excitation pulse to match the given phase velocity of the spin waves, one can excite different spin waves that match the dispersion curve, as one can see in Fig. 1, b. It is worth noting that, at high pulse velocities, it is possible to excite spin waves with extremely large wavevectors that cannot be excited within standard excitation methods such as microstip antennas due to a rapid drop of the antenna excitation efficiency for large wavevectors [15]. 




Fig. 2. Normalized excitation efficiency of spin waves with a conventional microstrip antenna (solid line) and via SCE with different pulse widths (points)
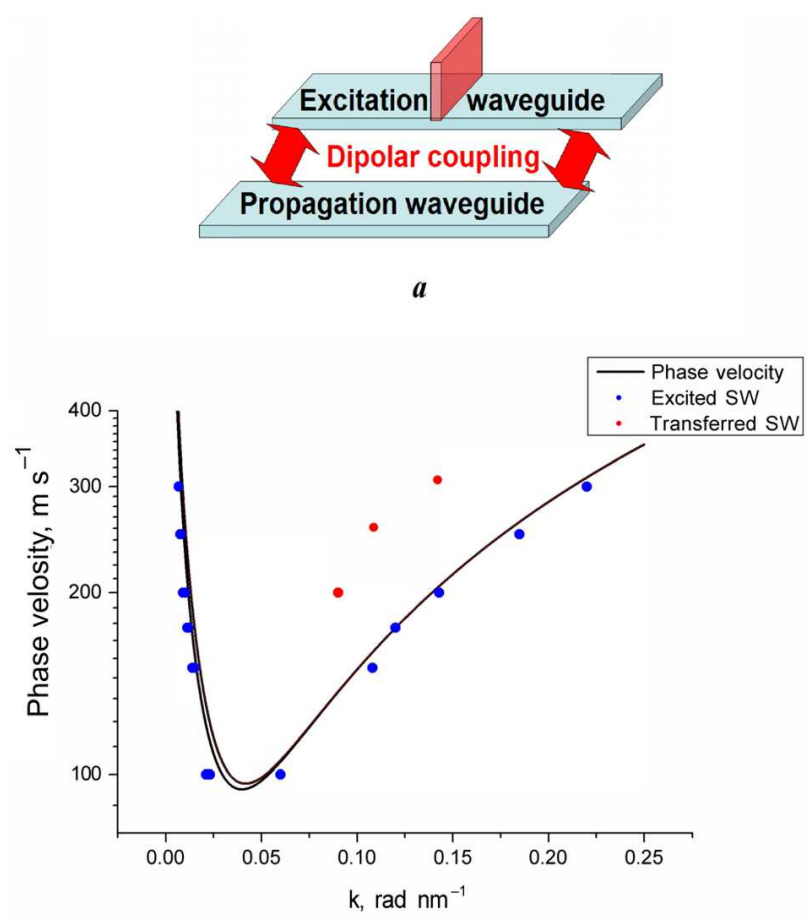

\section{b}

Fig. 3. SCE in coupled YIG waveguides. Schematic picture showing the excitation model with dipolarly coupled excitation and propagation waveguides $(a)$. Modeling results $(b)$. Solid line - theory, points - modeling for different velocities of the excitation pulse. Blue points correspond to the excited spin waves, and red points to the transferred spin waves in the propagation waveguide. Note that, for $v_{\text {pulse }}<200 \mathrm{~m} / \mathrm{s}$, blue and red points perfectly overlap
To investigate the excitation efficiency of SCE, we have performed simulations for varrious excitation pulse widths and compared it with the conventional excitation by a microstrip antenna. The excitation efficiency in the last case is well known: $\sim \exp \left(-\left(k / k_{0}\right)^{4}\right)$, where $k_{0}=2 \pi / a$, and $a$ is the antenna width [15]. As one can see, this is a rapidly decaying function for $k>k_{0}$. Thus, the antenna excitation is not efficient for the excitation of shortwavelength spin waves. On the other hand, decreasing the antenna width to the nanometer range is also difficult due to the electric matching of the antenna with an excitation source.

For the spin wave excitation via SCE, we can obtain the amplitudes of excited spin waves directly from modeling data. However, for comparison with the antenna excitation, we will show this result in the normalized form. In the modeling, the maximum spin wave amplitude was obtained for a minimally possible pulse velocity, just when it reaches the "bottom" of the spin wave spectrum. Thus, all modeling data for different excitation pulse widths and speeds were normalized by this value. A similar procedure was done for the antenna excitation, by normalizing it to the maximum value. Then both graphs were plotted alongside in Fig. 2. Different points correspond to different pulse excitation widths, namely 10,50 , and $100 \mathrm{~nm}$. As one can see, the excitation efficiency almost does not depend on the excitation pulse width and is governed by the SCE mechanism. Comparing to the conventional antenna excitation method (Fig. 2, solid line) we can see that SCE allows for the excitation of much shorter spin waves. It is worth noting that the excitation efficiency for SCE also drops with the increase of the excited wavevector. Thus, one cannot increase the pulse velocity forever to excite sub-nanometers waves. This effect is connected with the exchange interaction playing a governing role for so short spin waves. The detailed investigation of this effect lies beyond the scope of the current work.

Now, let's consider a double waveguide system, where we separate the excitation and propagation waveguide. Such separation can be convenient for the applications of future spintronics devices. It is known that the planar waveguides placed sufficiently close to each other can be coupled via dipolar fields leading to the energy transfer between them [12] (Fig. 3,a). This coupling strength depends on the interwaveguide dis- 
tance and waveguide thickness. Thus, for this calculation, we will consider 50-nm-thick waveguides separated by a $30-\mathrm{nm}$ gap. All other parameters of the waveguide remain the same, as for the single waveguide excitation scheme investigated above. We can define the coupling length $L_{c}$ as a distance, on which the energy from one waveguide is transferred to another one.

It should be noted that the coupling of waveguides leads to a splitting of the lowest width SW mode of a single waveguide into symmetric (acoustic) and antisymmetric (optic) collective modes of the coupled waveguides [12]. The frequency separation between the symmetric and antisymmetric modes is $\Delta f=\left|f_{\mathrm{s}}-f_{\text {as }}\right|$.

The idea of the excitation scheme (Fig. 3, $a$ ) is the following: the moving field pulse excites spin waves via $\mathrm{SCE}$ in the excitation waveguide, and, due to the dipolar coupling between the waveguides, this wave is transferred into the propagation waveguide.

Results of the modeling are shown in Fig. 3, $b$. The double line corresponds to the SW splitting due to the coupling between the waveguides. The striking feature of the obtained results is that the spin waves with $k_{\text {cr }}>0.15 \mathrm{rad} / \mathrm{nm}$ are not directly transferred into the propagation waveguide. Instead, the spin waves with smaller wavevectors are excited in the propagation waveguide. For $k<k_{\mathrm{cr}}$, the exact spin wave transfer occurs. Such behavior can be explained considering the dipolar nature of the interwaveguide coupling. According to [12], the dependence of the normalized output power of the second waveguide $P_{2 \text { out }} /\left(P_{1 \text { out }}+P_{2 \text { out }}\right)$ can be expressed as:

$P_{\text {2out }} /\left(P_{\text {lout }}+P_{\text {2out }}\right)=\sin ^{2}\left(\pi L_{\mathrm{W}} /\left(2 L_{\mathrm{c}}\right)\right)$,

where $L_{\mathrm{c}}$ is the coupling length, and $L_{\mathrm{W}}$ is the length of the coupled waveguides. The coupling length can be calculated as

$L_{\mathrm{c}}=\frac{v_{\mathrm{gr}}}{2 \Delta f}$,

where $v_{\text {gr }}$ is the group velocity in the isolated waveguide, and $\Delta f$ is the frequency separation.

The dependence of the normalized output power (1) on the spin wave wavevector is shown on Fig. 4. As one can see, it has the oscillatory behavior and approaches to zero at $k>k_{\mathrm{cr}}$. This fact indicates that the dipolar coupling is not efficient for the transfer

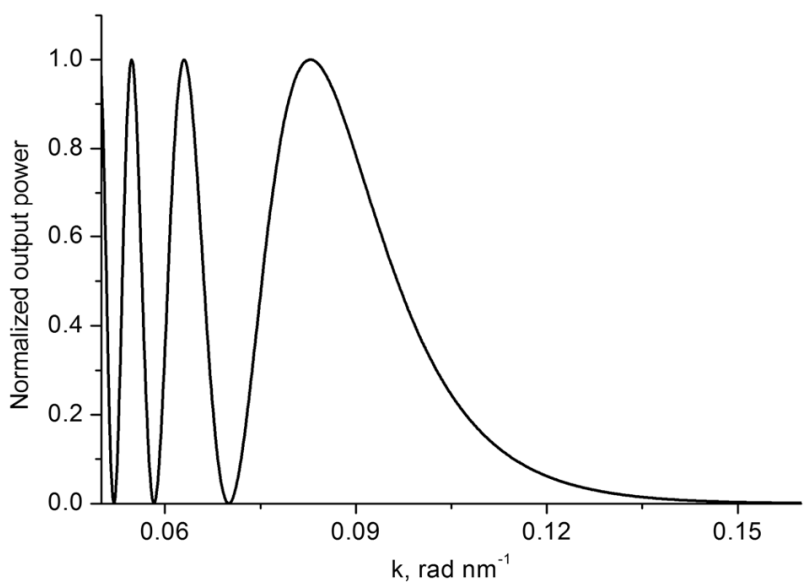

Fig. 4. Normalized output power dependence on the spin wave wavevector for coupled waveguides

of ultrashort spin waves excited in the first waveguide. Nevertheless, the modeling shows that some SW transfer still occurs (see Fig. 3, b). This effect will be investigated in the following works. A noteworthy feature of the dipolar coupling is that it has zero transmission regions even for $k<k_{\mathrm{cr}}$ (for example, at $k=0.07 \mathrm{rad} / \mathrm{nm})$. Unfortunately, the limited number of modeling points do not allow us to investigate the SW transfer around these points more precisely. This will be executed in the following works.

Now, we briefly discuss how one can obtain such moving pulses for the spin wave excitation. The simplest way is to use the double waveguide geometry considered above. One of the possible ground states is the antiparallel one with a domain wall in one of the waveguides (see Fig. 6, $L$ in [12]). Applying an electric current or magnetic field, one can move this domain wall with various speeds, which causes the excitation of spin waves via SCE. Then, as was shown above, those SW can be transferred to the adjacent waveguide via the dipolar interaction.

\section{Conclusions}

We have demonstrated that SCE can be effectively used to excite ultrashort spin waves in a magnetic waveguide system. The moving magnetic field pulse with different pulse widths can be used as a source of such excitation. Varying the excitation pulse speed can be used to tune the excited SW wavelength. The typically excited SW wavelength in the performed modeling was about $\lambda \sim 40 \mathrm{~nm}$, but the excitation of 
shorter waves was also possible. It is shown that the excitation efficiency decreases with an increase of the wave vectors of excited waves. The dipolarly coupled waveguide system can also be used for the SW excitation, in this case, the spin waves excited in the first waveguide are transferred to the second one via the dipolar coupling. However, this mechanism has some limitation and do not support the transfer of ultrashort spin waves. The obtained results are important for the emerging field of magnonics and future applications in different devices utilizing ultrashort spin waves for the signal processing.

This work was supported in part by grant 18BF052$01 M$.

1. A. Haldar, D. Kumar, A.O. Adeyeye. A reconfigurable waveguide for energy-efficient transmission and local manipulation of information in a nanomagnetic device. Nat. Nanotech. 11, 437 (2016).

2. K. Wagner, A. Ka'kay, K. Schultheiss, A. Henschke, T. Sebastian, H. Schultheiss. Magnetic domain walls as reconfigurable spin-wave nanochannels. Nat. Nanotech. 11, 432 (2016).

3. V.E. Demidov, S. Urazhdin, S.O. Demokritov. Direct observation and mapping of spin waves emitted by spintorque nano-oscillators. Nature Materials, 9, 984 (2010).

4. M. Madami, S. Bonetti, G. Consolo, S. Tacchi, G. Carlotti, G. Gubbiotti, F.B. Mancoff, M.A. Yar, J. Akerman. Direct observation of a propagating spin wave induced by spintransfer torque. Nat. Nanotech. 6, 635 (2011).

5. C.S. Davies, A. Francis, A.V. Sadovnikov, S.V. Chertopalov, M.T. Bryan, S.V. Grishin, D.A. Allwood, Y.P. Sharaevskii, S.A. Nikitov, V.V. Kruglyak. Towards gradedindex magnonics: Steering spin waves in magnonic networks. Phys. Rev. B 92, 020408(R) (2015).

6. V.E. Demidov, M.P. Kostylev, K. Rott, J. Münchenberger, G. Reiss S.O. Demokritov. Excitation of short-wavelength spin waves in magnonic waveguides. Appl. Phys. Lett. 99, 082507 (2011).

7. Y. Au, E. Ahmad, O. Dmytriiev, M. Dvornik, T. Davison, V.V. Kruglyak. Resonant microwave-to-spin-wave transducer. Appl. Phys. Lett. 100, 182404 (2012).

8. H. Yu, G. Duerr, R. Huber, M. Bahr, T. Schwarze, F. Brandl, D. Grundler. Omnidirectional spin-wave nanograting couple. Nat. Commun. 4, 2702 (2013).

9. G. Dieterle, J. Förster, H. Stoll, A.S. Semisalova, S. Finizio, A. Gangwar, M. Weig, M. Noske, M. Fähnle, I. Bykova, J. Grafe, D.A. Bozhko, Yu. Musiienko-Shmarova, V. Tiberkevich, A.N. Slavin, C.H. Back, J. Raabe, G. Schütz, S. Wintz. Coherent excitation of heterosymmetric spin waves with ultrashort wavelengths. Phys. Rev. Lett. 122, 117202 (2019).
10. M. Yan, C. Andreas, A. Ka'kay, F. Garcia-Sanchez, R. Hertel. Fast domain wall dynamics in magnetic nanotubes: Suppression of Walker breakdown and Cherenkov-like spin wave emission. Appl. Phys. Lett. 99, 122505 (2011).

11. M. Yan, C. Andreas, A. Ka'kay, R. Hertel. Spin-Cherenkov effect and magnonic Mach cones. Phys. Rev. B 88, 220412(R) (2013).

12. Q. Wang, P. Pirro, R. Verba, A. Slavin, B. Hillebrands, A.V. Chumak. Reconfigurable nanoscale spin-wave directional coupler. Sci. Adv. 4(1), e1701517 (2018).

13. A.A. Serga, A.V. Chumak, B. Hillebrands. YIG magnonics. J. Phys. D: Appl. Phys. 43, 264002 (2010).

14. M.J. Donahuem, D.G. Porter. Interagency Report NISTIR 6376 (National Institute of Standards and Technology, 1999).

15. A.G. Gurevich, G.A. Melkov. Magnetization Oscillations and Waves (CRC Press, 1996).

16. J. Xia, X. Zhang, M. Yan, W. Zhao, Y. Zhou. SpinCherenkov effect in a magnetic nanostrip with interfacial Dzyaloshinskii-Moriya interaction. Sci. Rep. 6, 25189 (2016).

17. T. Brächer, P. Pirro, B. Obry, B. Leven, A.A. Serga, B. Hillebrands. Mode selective parametric excitation of spin waves in a $\mathrm{Ni}_{81} \mathrm{Fe}_{19}$ microstripe. Appl. Phys. Lett. 99, 162501 (2011).

18. O.V. Prokopenko, D.A. Bozhko, V.S. Tyberkevych, A.V. Chumak, V.I. Vasyuchka, A.A. Serga, O. Dzyapko, R.V. Verba, A.V. Talalaevskij, D.V. Slobodianiuk, Yu.V. Kobljanskyj, V.A. Moiseienko, S.V. Sholom, V.Yu. Malyshev. Recent trends in microwave magnetism and superconductivity. Ukr. J. Phys. 64, 888 (2019).

Received 28.05.20

\section{Д.В. Слободянюк}

\section{ЗБУДЖЕННЯ УЛЬТРАКОРОТКИХ СПІНОВИХ ХВИЛЬ ЗА ДОПОМОГОЮ СПІНОВОГО ЕФЕКТУ ЧЕРЕНКОВА В МАГНІТНИХ ХВИЛЕВОДАХ}

У роботі проаналізовано збудження ультракоротких спінових хвиль за допомогою спінового ефекту Черенкова методами мікромагнітного моделювання. Запропонована схема збудження хвиль є відносно простою та легко перебудовується. Отримано ефективність збудження спінових хвиль для різних швидкостей та ширин збуджуючого імпульсу. Розглянуто систему двох зв'язаних хвилеводів. В цьому випадку спінові хвилі збуджуються в першому хвилеводі та переносяться у другий через дипольний зв'язок між хвилеводами. Також показано, що збудження та передача спінових хвиль в такій системі має певні обмеження, пов'язані з дипольними механізмами у хвилеводах.

Ключов $i$ слова: спіновий ефект Черенкова, магнітний хвилевод, спінова хвиля, спінтроніка.

ISSN 2071-0194. Ukr. J. Phys. 2021. Vol. 66, No. 5 